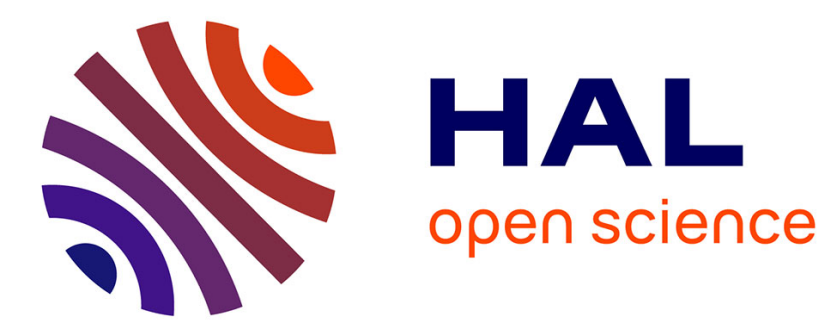

\title{
Tunnel résonnant et effets d'électrons chauds dans les structures à double barrière: synthèse
}

D. Lippens, L. de Saint Pol, R. Bouregba, P. Mounaix, T. Vinchon

\section{To cite this version:}

D. Lippens, L. de Saint Pol, R. Bouregba, P. Mounaix, T. Vinchon. Tunnel résonnant et effets d'électrons chauds dans les structures à double barrière: synthèse. Revue de Physique Appliquée, 1989, 24 (1), pp.17-30. 10.1051/rphysap:0198900240101700 . jpa-00246022

\section{HAL Id: jpa-00246022 https://hal.science/jpa-00246022}

Submitted on 1 Jan 1989

HAL is a multi-disciplinary open access archive for the deposit and dissemination of scientific research documents, whether they are published or not. The documents may come from teaching and research institutions in France or abroad, or from public or private research centers.
L'archive ouverte pluridisciplinaire HAL, est destinée au dépôt et à la diffusion de documents scientifiques de niveau recherche, publiés ou non, émanant des établissements d'enseignement et de recherche français ou étrangers, des laboratoires publics ou privés. 
Classification

Physics Abstracts

$85.30 \mathrm{M}-72.20$

\title{
Tunnel résonnant et effets d'électrons chauds dans les structures à double barrière : synthèse
}

\author{
D. Lippens, L. De Saint Pol, R. Bouregba, P. Mounaix et T. Vinchon \\ Centre Hyperfréquences et Semiconducteurs, U.A. 287 C.N.R.S., bâtiment P4, U.F.R. d'I.E.E.A., \\ Université des Sciences et Techniques de Lille Flandres Artois, 59655 Villeneuve d'Ascq Cedex, France
}

(Reçu le $1^{\text {er }}$ juillet 1988, accepté le 12 septembre 1988)

\begin{abstract}
Résumé. - Des progrès remarquables ont été faits dans les caractéristiques des doubles barrières à tunnel résonnant en raison des améliorations des techniques d'épitaxie des structures à modulation de matériau. Ceci a été à l'origine de très nombreuses études tant sur les propriétés dynamiques et statiques que sur de nouveaux composants. Dans cet article, nous nous proposons de faire le point sur ce sujet, sur la base des études physiques, permettant de comprendre, de caractériser et d'optimiser les hétérostructures à double barrière. Nous montrons que, relativement aux applications composants, il est nécessaire de prendre en compte des aspects plus spécifiques tels que la réaction de charge d'espace. Enfin, nous considérons les effets d'électrons chauds, souvent indissociables des effets tunnel.

Abstract. - Remarkable progress has been made in the characteristics of resonant tunneling double barrier due to recent advancements in epitaxy techniques of modulated semiconductor structures. Much interest has been generated in studying the static and dynamic properties as well as proposing novel devices. This paper reviews this subject area on the basis of physical studies allowing us to understand characterize and lastly optimize double barriere heterostructures. We show that, in device applications, more specific aspects such as space charge reaction has to be taken into account. Lastly, it is pointed out that hot electron effects are often combined with tunneling effect and cannot be ignored.
\end{abstract}

\section{Introduction.}

Pour une structure à puits quantique créée par deux barrières de potentiel de faibles épaisseurs (typiquement entre $20 \AA$ et $50 \AA$ ) on met en évidence un effet tunnel résonnant qui se traduit au niveau des caractéristiques électriques par l'existence d'une résistance différentielle négative (NDR). L'analyse de ce phénomène ainsi que sa démonstration expérimentale date des années 1970 [1, 2]. Cependant, les difficultés de réalisation technologique d'alors ont fait que de telles structures ont été peu développées jusqu'en 1983 où les travaux de Sollner ont montré la possibilité d'utiliser ces effets jusqu'à $2,5 \mathrm{THz}$ [3]. Ces résultats très novateurs combinés à la maîtrise actuelle des techniques d'épitaxie ont suscité un regain d'intérêt formidable sur ce sujet tant théorique qu'expérimental. La motivation essentielle de ces études est double. Tout d'abord, l'inertie de l'effet de tunnel résonnant devrait se situer pour des structures optimisées à l'échelle de la dixième de picoseconde, du fait de la combinaison des temps de passage dans les barrières et de piégeage dans le puits. C'est donc un effet physique dont le temps de réponse est théoriquement un ordre de grandeur en dessous de ceux de phénomènes physiques plus conventionnels (inertie d'avalanche, de transit...). Ensuite, il devrait être possible d'obtenir, en raison de la sélectivité du phénomène, un rapport entre courant maximum $J_{\text {pic }}$ et courant minimum ( $\left.J_{\text {vallée }}\right)$ très important. La figure 1 illustre ce dernier point en retraçant au cours des années les améliorations apportées sur le contraste de courant. Depuis la démonstration en 1985 puis en 1986 [4, 5] par Sakaki d'une NDR significative à température ambiante sur des structures à double barrière $\mathrm{AlAs} / \mathrm{GaAs}$, le rapport $J_{\text {pic }}$ sur $J_{\text {vallée }}$ n'a cessé d'augmenter. Les valeurs les plus importantes sont proches de 4 pour des structures $\mathrm{GaAlAs} / \mathrm{GaAs}$ [6], de 6 pour les matériaux InAlAs/InGaAs [7, 8]. La fabrication de structures contraintes InGaAs/AlAs a porté ce rapport à 14 [9] très récemment.

Parallèlement et grâce à ces études de matériaux, de nombreuses propositions de composants nou- 


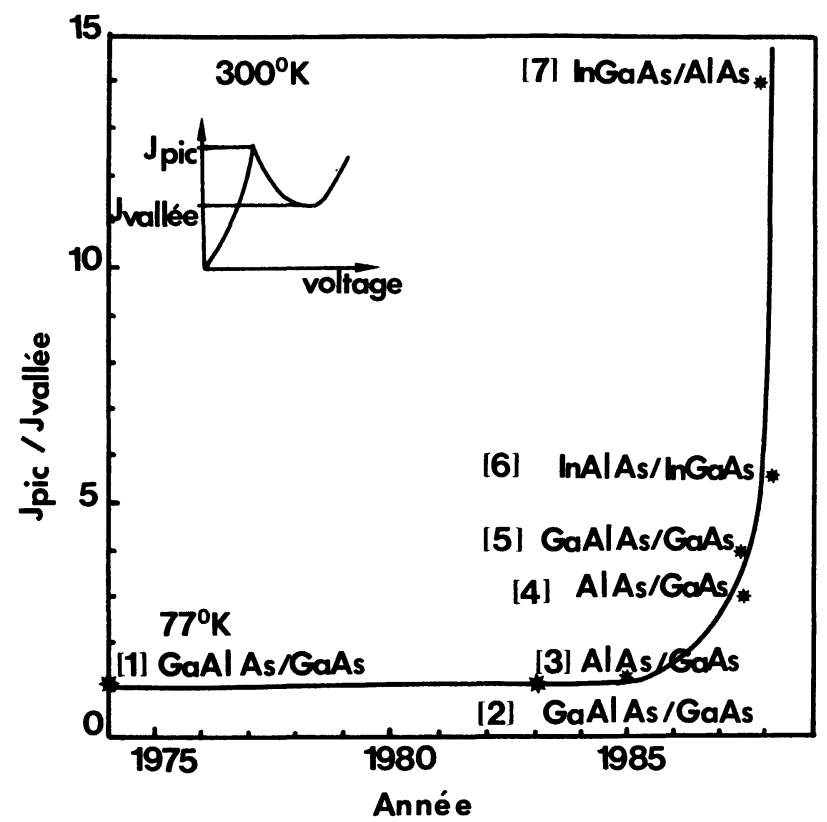

Fig. 1. - Evolution du rapport $J_{\text {pic }} / J_{\text {vallee }}$ au cours des dernières années.

[Improvement of $J_{\text {peak }} / J_{\text {valley }}$ ratio during past few years.]

veaux intégrant des structures à double barrière sont parues dans la littérature. Elles concernent tant les dipôles avec pour objectif principal la génération d'ondes hyperfréquences $[10,11]$ aux fréquences millimétriques et submillimétriques que les tripôles dont les applications visent plus particulièrement la logique rapide $[12,13]$.

Dans cet article, nous nous proposons de faire le point sur ce passage étude physique matériau-application composant. Nous verrons que si le fonctionnement des structures à tunnel résonnant est simple dans son principe il est nécessaire de calculer puis d'analyser les caractéristiques électriques si l'on veut optimiser le matériau et la structure. Ces aspects seront traités dans les paragraphes 2,3 et 4 . Les problèmes de temps de réponse permettant d'appréhender le régime dynamique et de réaction de charge d'espace qu'on ne peut négliger dans un fonctionnement à courant élevé, seront abordés dans le paragraphe 5 . Les caractérisations physique et électrique des structures à doubles barrières utilisables directement dans les applications composants seront traitées dans le paragraphe 6 . Ce sont ces mêmes applications qui seront développées dans le paragraphe 7 .

\section{Principe de fonctionnement-outil numérique.}

2.1 STRUCTURE. - La structure semiconductrice à tunnel résonnant la plus élémentaire est donnée figure 2. L'hétérostructure réalisée de cette façon présente des discontinuités de bande de conduction et de valence suivant la direction de croissance.

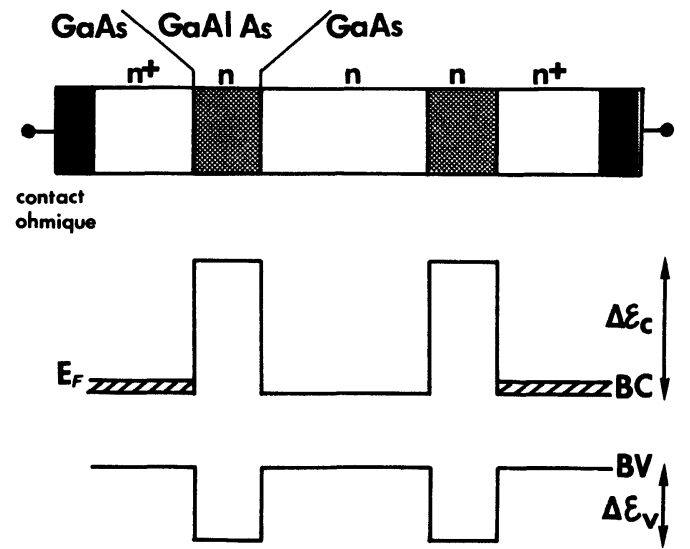

Fig. 2. - Structure schématisée de la double barrière.

[Schematic double barrier heterostructure (DBH).]

Ainsi un électron se déplaçant sous l'effet d'une polarisation extérieure doit traverser successivement par effet tunnel les zones classiquement interdites (barrières de potentiel). Enfin, pour permettre la réalisation de contacts ohmiques, on insère l'ensemble puits-barrières constituant la zone active entre deux zones fortement dopées. Ceci conduit au profil de potentiel schématisé sur cette même figure, les zones $\mathrm{n}^{+}$étant dégénérées.

Le principe de fonctionnement peut se comprendre à l'aide de la figure 3 . En absence de polarisation, les porteurs piégés dans le puits de potentiel ont leur énergie quantifiée en raison de leur confinement. Les barrières de potentiel sont minces et il en résulte que la probabilité de passage par effet tunnel à travers les barrières n'est plus négligeable. Nous parlerons dans ce cas d'états quasi liés par opposition aux exemples de confinement strict dans la direction de croissance (c'est ce dernier cas dans la plupart des applications TEGFET). Sous l'action d'une polarisation extérieure, il est possible d'aligner le niveau de Fermi d'une des zones conductrices d'accès au premier niveau discret du puits. Cet ajustement de l'énergie des porteurs s'apprêtant à franchir la double barrière à celle du puits se traduit par une augmentation très importante de la transmission $T$ (effet de résonance). A plus forte tension les niveaux d'énergie se désalignent entraînant dans le cas idéal d'une population électronique peu dispersée en énergie une chute brutale des valeurs de $T(\mathrm{c})$. Des pics successifs peuvent ainsi être observés à chaque niveau d'énergie discret du puits quantique (d).

2.2 MÉthode D’ANALYSE. - La figure 4 schématise les situations rencontrées en pratique quant aux variations de potentiel vues par les électrons au cours de leur déplacement. La figure a correspond à un état de quasi équilibre. C'est ainsi le cas lorsque la structure double barrière est insérée dans la base très fortement dopée d'un transistor. La mise en résonance de la structure est ici obtenue par variation 

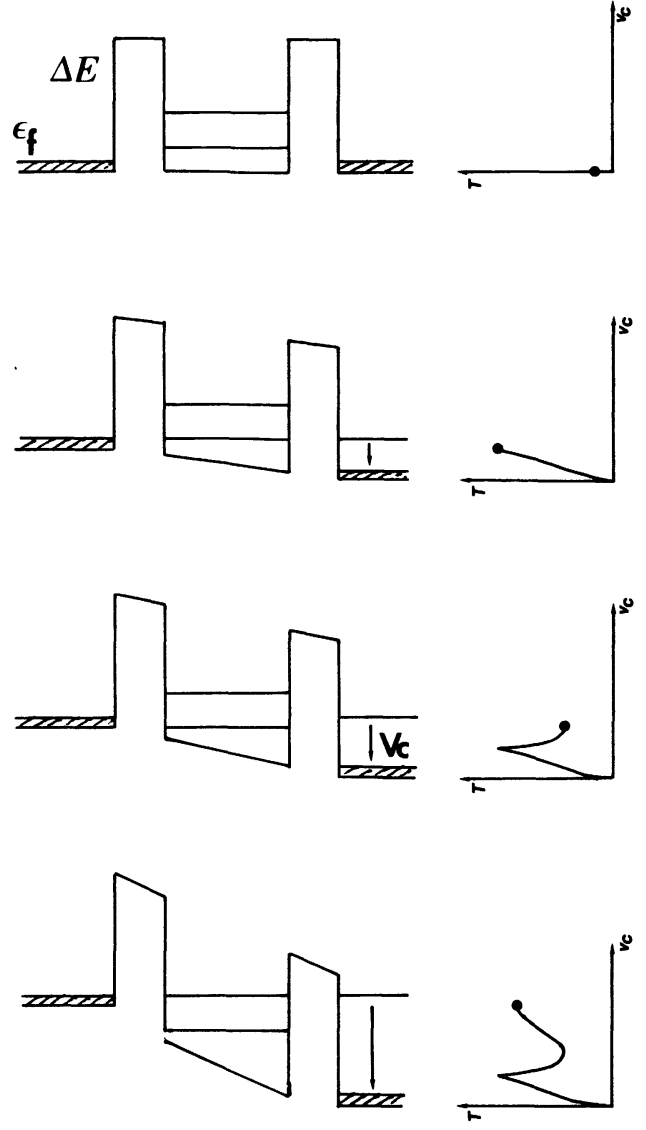

Fig. 3. - Principe de fonctionnement des structures à double barrière.

[Operating principle of $\mathrm{DBH}$.

de l'énergie des porteurs injectés. Le calcul de la fonction d'onde et de la transmission est alors faisable analytiquement [14] et permet une première approche du problème. Les figures $b$ et $c$ correspondent au cas d'une mise en résonance par application de tension extérieure pour des densités de courant faibles. Bien que rigoureusement les probabilités de présence soient calculées dans ce cas à l'aide des fonctions d'Airy il est possible d'introduire l'approximation marche plate proposée dans l'article original d'Esaki [1] qui constitue nous le verrons une excellente approximation. Enfin la figure d illustre le cas où la réaction de charge d'espace est importante entraînant une modification significative du profil de potentiel.

Plusieurs formalismes peuvent être utilisés pour calculer la caractéristique de transmission en régime statique et en supposant que le passage de la structure puits barrière par les porteurs se fait de façon cohérente. Dans ce cas, aucun terme de perturbation dû aux interactions sur phonons n'est pris en compte. Ultérieurement nous discuterons de cette approximation.

A titre d'exemple citons les formalismes de Tsu [1] (approximation marche plate) de Ricco et al. [15]
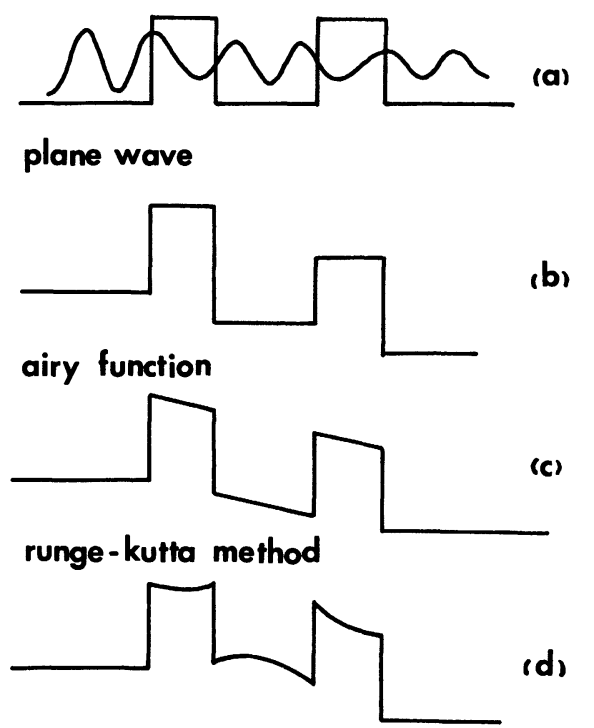

Fig. 4. - Variation spatiale du potentiel vu par les porteurs. (a) en absence de tension appliquée ; (b) approximation marche plate ; (c) potentiel tilté ; (d) pour une réaction de charge d'espace importante.

[Potential-energy diagram of DBH. (a) without bias voltage ; (b) flat potential approximation ; (c) titled potential step ; (d) band bending due to space charge reaction.]

(matrice de transfert) de Vassel et al. [16] (Schrödinger par Runge Kutta). L'accès à l'information de caractéristique courant-tension est obtenu de la façon suivante :

la résolution de Schrödinger permet le calcul des fonctions d'onde dans chaque région de potentiel d'où l'on déduit le paramètre de transmission [14]. La fonction d'onde longitudinale (suivant la direction de croissance) satisfait à l'équation unidimensionnelle :

$$
\begin{aligned}
-\frac{\hbar^{2}}{2} \frac{\mathrm{d}}{\mathrm{d} z}\left(\frac{1}{m^{*}} \frac{\mathrm{d} \Psi}{\mathrm{d} z}\right)+V(z) \Psi\left(k_{z}, z\right) & = \\
& =\varepsilon_{z} \Psi\left(k_{z}, z\right)
\end{aligned}
$$

où $V(z)$ résulte de la superposition du potentiel cristallin et électrique.

Le courant dans la structure est obtenu à partir de l'équation [17] :

$$
\begin{aligned}
J=\frac{k T q m^{*}}{2 \pi^{2} \hbar^{3}} \int_{0}^{\infty} T(\varepsilon) \times \\
\quad \times \ln \left(\frac{1+\exp \left[\left(\varepsilon_{\mathrm{F}}-\varepsilon_{z}\right) / k T\right]}{1+\exp \left[\left(\varepsilon_{\mathrm{F}}-\varepsilon_{z}-V_{\mathrm{c}}\right) / k T\right]}\right) \mathrm{d} \varepsilon .
\end{aligned}
$$

Transmission. - La figure 5 donne le résultat d'un calcul de transmission effectué pour une variation linéaire du potentiel lorsque l'on fait varier la tension appliquée $V_{\text {collecteur }}$. Le point important sur cette figure est la diminution notable du pic de transmission à la résonance par rapport à la valeur 


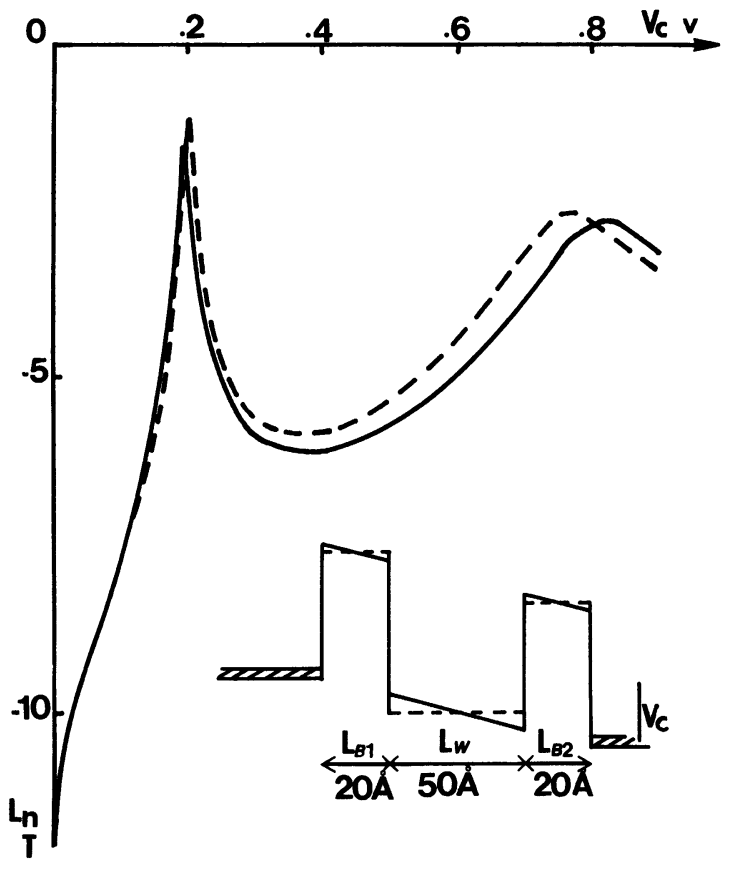

Fig. 5a. - Logarithme népérien de la transmission en fonction de la tension appliquée $V_{\mathrm{c}}$ (potentiels plats et tiltés).

[Natural log of the transmission coefficient versus the applied voltage $V_{c}$ (flat and titled potential steps).]

optimale $T_{\text {rés }}=1$. Ricco et al . ont montré très clairement que cette diminution résulte de la destruction de la symétrie de transparence entre les barrières gauche et droite [15]. Nous verrons que ce résultat motive les études d'optimisation des structures dissymétriques. Par ailleurs, pour des valeurs importantes de la tension $V_{c}$, bien que l'énergie des électrons s'apprêtant à franchir la deuxième barrière devienne supérieure à la hauteur de la barrière de potentiel, on note une diminution des valeurs de la transmission. Cet effet s'explique par le phénomène de réflexion quantique qui maintient les phénomènes de résonance pour des valeurs d'énergie supérieures à la hauteur de potentiel de la deuxième barrière. Enfin, on constate que la solution obtenue en marche plate donnée en comparaison constitue une très bonne approximation pouvant être utilisée dans les études systématiques.

La figure $5 b$ donne un exemple de calcul de la transmission lorsque l'on maintient constante la tension collecteur et que l'on fait varier l'énergie des porteurs injectés. En pratique ce cas correspond à un fonctionnement tripôle où la variation de l'énergie des électrons dans l'émetteur est obtenue électroniquement. On constate, comme cela était prévisible, que l'énergie de résonance est minorée d'environ la moitié de la tension $\left(\varepsilon_{\mathrm{r}}^{\prime}=\varepsilon_{\mathrm{r}}(V=0)-\right.$ $\left.V_{\mathrm{c}} / 2\right)$.

Fonction d'ondes. - La figure 6a montre les résultats de calcul de fonction d'onde (partie réelle

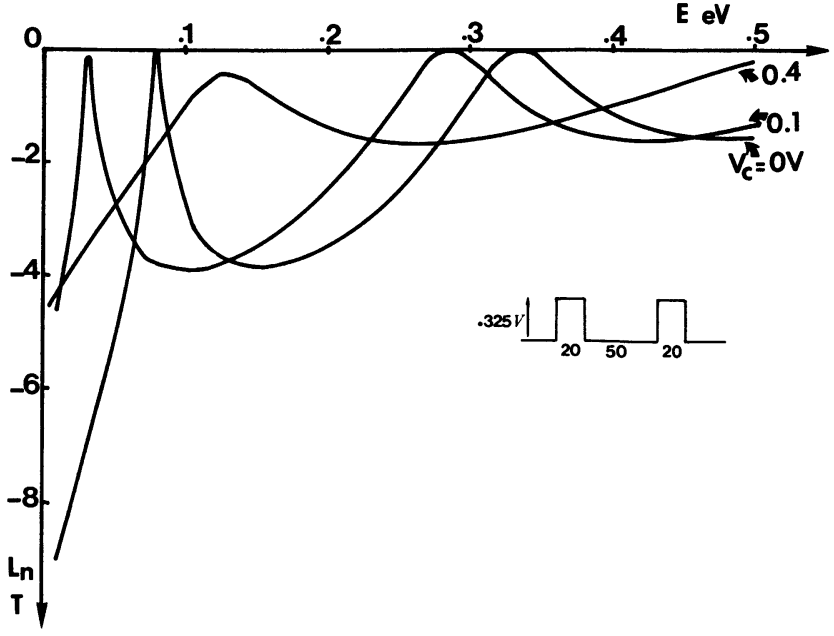

Fig. 5b. - $\ln T$ en fonction de l'énergie d'injection pour une tension appliquée de $0 \mathrm{~V} ; 0,1 \mathrm{~V} ; 0,4 \mathrm{~V}$.

[ $\ln T$ versus energy at $V_{\mathrm{c}}=0 \mathrm{~V}, 0.1 \mathrm{~V}$ and $0.4 \mathrm{~V}$.]

et imaginaire) ainsi que la probabilité de présence, à l'équilibre, respectivement pour une énergie incidente correspondant à la $1^{\text {re }}$ résonance et à la $2^{\mathrm{e}}$ résonance. On constate une pénétration importante des probabilités de présence électronique dans les
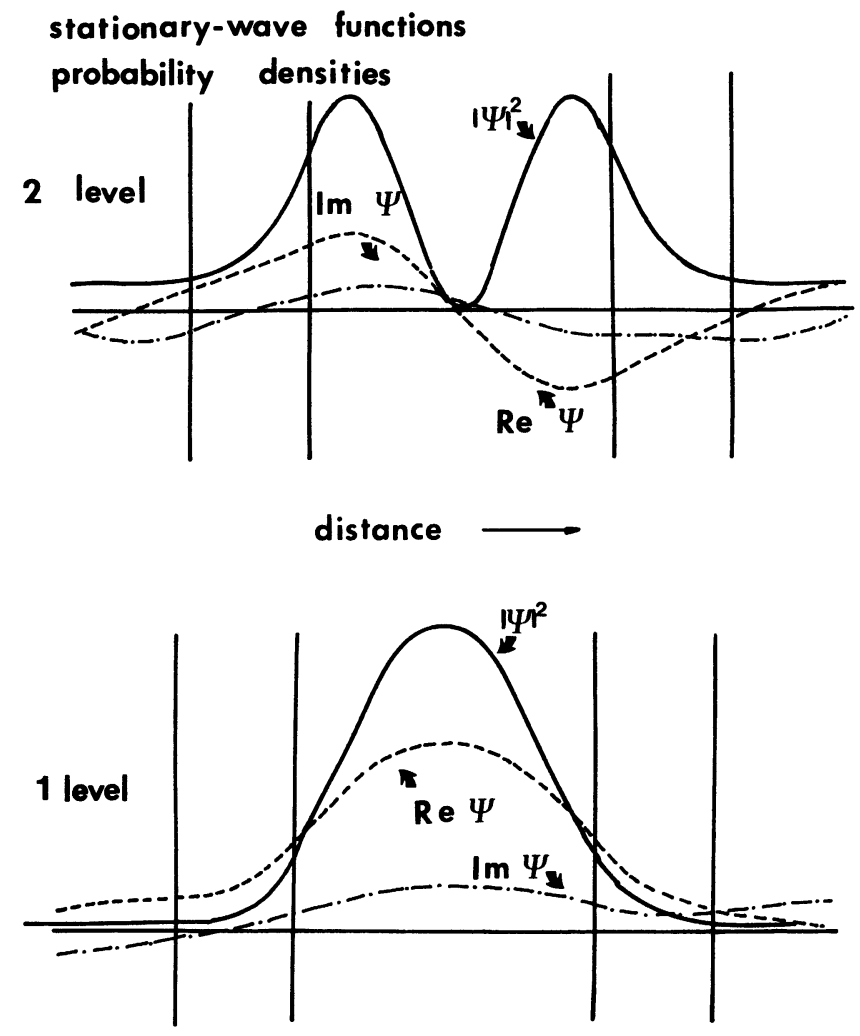

Fig. 6a. - Fonction d'onde et probabilité de présence à la première et seconde résonance (variation de l'énergie d'injection).

[Wave-functions and probability at first and second resonance at equilibrium.] 
barrières de potentiel pour ces deux états d'énergie. Outre la possibilité de délocalisation des électrons déjà évoquée, ceci a pour conséquence que la condition de résonance diffère de façon significative de celle du puits infini. Rappelons que dans ce dernier cas il y a adaptation entre largeur du puits quantique et demi-longueur d'onde associée aux électrons.

La figure $6 \mathrm{~b}$ montre un exemple de calcul de fonction d'onde pour une tension de polarisation appliquée correspondant à une transmission proche de la résonance et hors résonance. Pour maintenir les probabilités de présence hors résonance à des valeurs suffisantes, les résultats sont présentés pour une amplitude d'onde transmise normalisée à l'unité. Deux points importants peuvent ici être observés. On note tout d'abord un décalage progressif de la probabilité de présence électronique qui conduit à couplage plus important avec le contact de collecteur. La transparence de la deuxième barrière est alors élevée. Ensuite on constate que pour la situation hors résonance, la probabilité de présence est très élevée en amont de la $1^{\text {re }}$ barrière. Ceci correspond à la désadaptation de transmission établie dans ce cas.
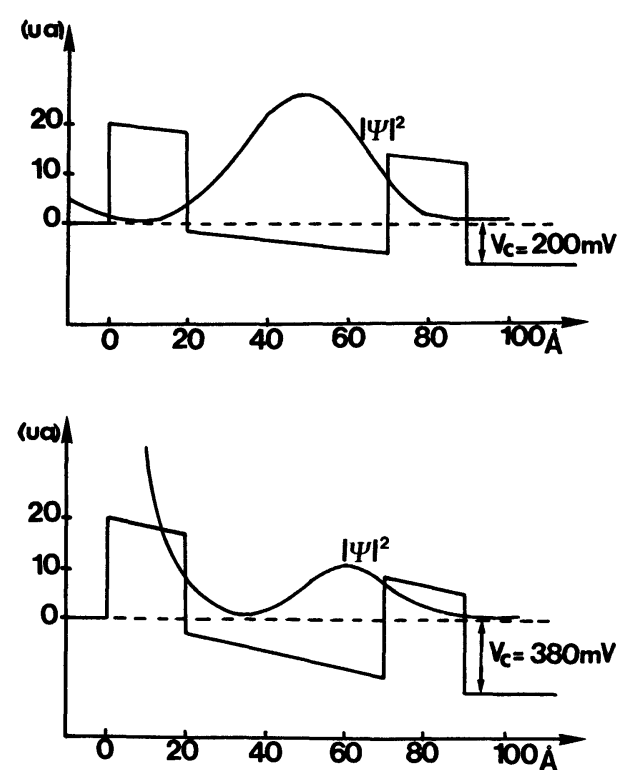

Fig. 6b. - Fonction d'onde pour des tensions de polarisation proche de la résonance et hors résonance.

[Wave function at and off resonance.]

\section{Choix d'une géométrie.}

3.1 ETATS QUASI LIÉS. - La figure 7 montre comment varie la fonction de transmission pour une variation de l'énergie des porteurs incidents à différentes largeurs de puits quantiques. L'épaisseur de barrière est maintenue constante $L_{\mathrm{B}}=20 \AA$. Les

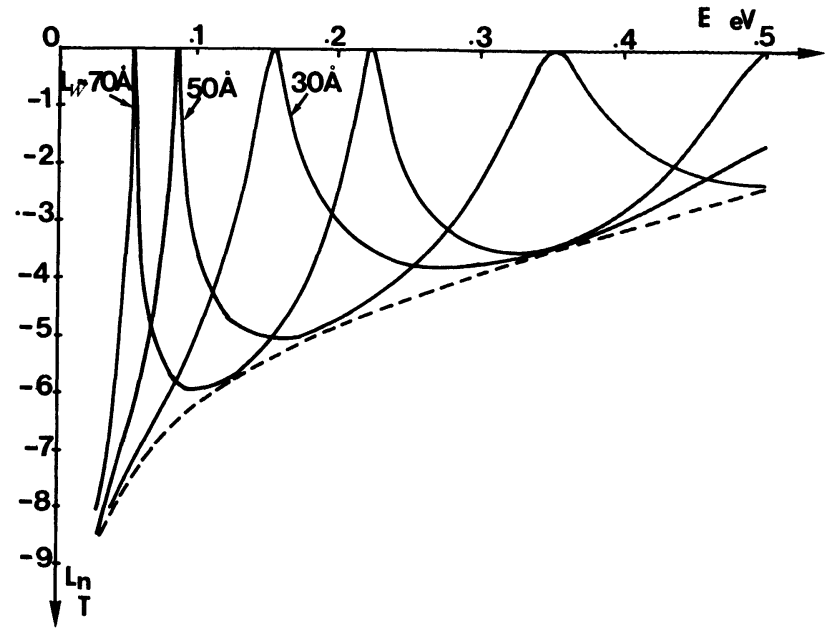

Fig. 7. - Energie de résonance en fonction de la largeur de puits quantique.

[Resonant energy versus quantum well width.]

énergies de résonance $\varepsilon_{\mathrm{r}}$ correspondant aux maximums de transmission, augmentent au fur et à mesure que $L_{\mathrm{w}}$ diminue. On peut montrer par étude systématique que $\varepsilon_{\mathrm{r}}$ varie en $1 / L_{\mathrm{w}}$ et $1 / L_{\mathrm{w}^{2}}$ suivant que la localisation est plus ou moins importante. Par ailleurs, le lieu de valeurs de $T_{\text {hors }_{\text {rés }}}$ en fonction de l'énergie est comparable à la transmission d'une seule barrière d'épaisseur $2 L_{\mathrm{B}}$. Ceci est conforme à l'analyse de Ricco. $T_{\text {hors }_{\text {res }}} \sim T_{\mathrm{g}} T_{\mathrm{d}}$, ces deux grandeurs désignant les transmissions des barrières gauche et droite. Une information supplémentaire que l'on peut tirer des résultats concerne la largeur de la résonance $\left(\Gamma_{\mathrm{e}}\right)$, celle-ci étant d'autant plus sélective que $L_{\mathrm{w}}$ est grand. On peut montrer que $\Gamma_{\mathrm{e}}$ varie en $1 / L_{\mathrm{w}}[18]$.

La figure $\$ 8$ montre les variations des états quasi liés en fonction de la largeur de barrière $L_{\mathrm{B}}$ pour $L_{\mathrm{w}}=50 \AA$. Pour $L_{\mathrm{B}}>20 \AA$ le niveau de résonance est quasi constant. Au-dessous de cette valeur critique, $\varepsilon_{\mathrm{r}}$ décroît du fait d'un couplage avec le continuum de plus en plus important. Ces résultats sont en accord avec ceux publiés par Badher [19].

\subsection{CONTRASTE DE TRANSMISSION ET DE COURANT.}

La figure 9 montre les variations du rapport $T_{\text {rés }} / T_{\text {hors }_{\text {rés }}}$ en fonction des caractéristiques géométriques $L_{\mathrm{w}}$ et $L_{\mathrm{B}}$. Le contraste de résonance décroît exponentiellement lorsque l'on diminue la largeur de barrière de potentiel. Ce résultat peut être interprété qualitativement par analogie avec une cavité résonnante électromagnétique. On trouve ainsi étendu au cas des semi-conducteurs, le fait qu'en augmentant le couplage de la cavité avec l'extérieur (augmentation de la transparence des barrières) on diminue la sélectivité. De façon plus quantitative il est possible d'interpréter ce résultat à 


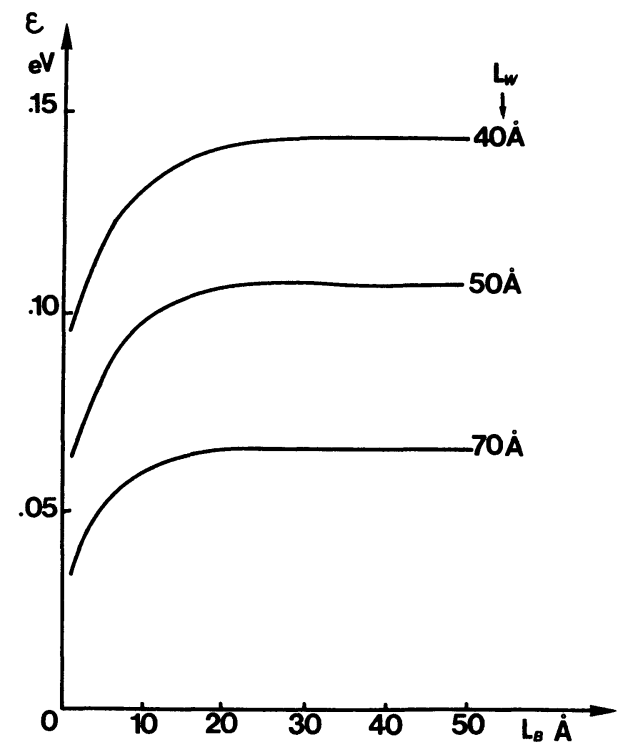

Fig. 8. - Energie de résonance en fonction de la largeur de barrière.

[Resonant energy versus barrier width.]

partir de l'analyse de Ricco qui conduit à l'expression approchée

$$
\frac{T_{\text {rés }}}{T_{\text {hors res }}} \sim \frac{1}{T^{2}} \sim \mathrm{e}^{+4 \alpha L_{\mathrm{B}}}
$$

où $\alpha$ est le facteur d'atténuation de la fonction d'onde dans la barrière. L'évolution avec $L_{\mathrm{w}}$ est similaire. Les variations sont cependant moins importantes. La diminution du rapport $T_{\text {ress }} / T_{\text {hors }}$ lorsque $L_{\mathrm{w}}$ décroît s'explique en observant que $T_{\text {hors res }}$ augmente alors que $T_{\text {rés }}$ est quasi constant figure 7.

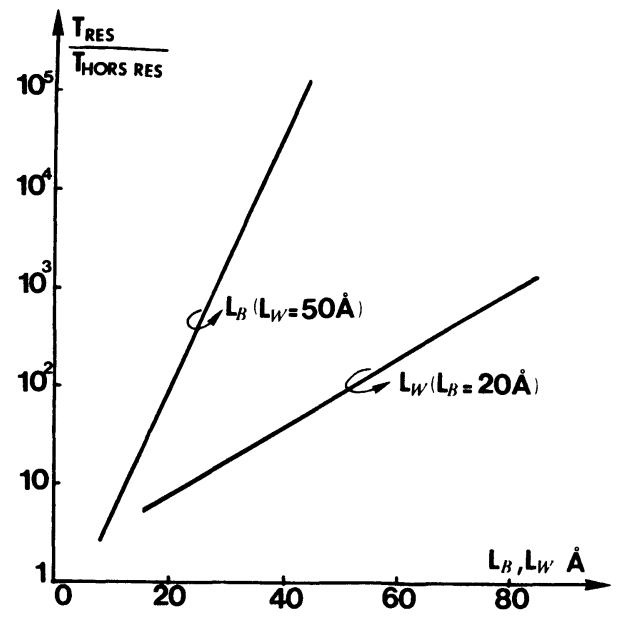

Fig. 9. - Contraste de transmission en fonction des largeurs de barrière et de puits quantique.

$\left[T_{\text {res }} / T_{\text {off }}\right.$ versus barrier and quantum well widths.]

En résumé, il est donc théoriquement possible d'obtenir des contrastes de transmission très élevés dont les valeurs atteignent rapidement 100 pour des largeurs de barrière de l'ordre de quelques couches atomiques (typiquement $20 \AA$ dans le système $\mathrm{GaAs} / \mathrm{GaAlAs})$. Le contraste de courant est bien moindre puisqu'il est possible d'établir que $J_{\text {pic }} / J_{\text {vallee }}$ est proportionnel à $1 / T$ et non à $1 / T^{2}$ [18]. Pour des structures réalisées technologiquement, d'autres phénomènes apparaissent tendant à diminuer le contraste de courant. Nous reviendrons sur ce point.

Le tableau I résume le sens des variations respectivement de $\varepsilon_{\mathrm{r}}$ (énergie de résonance), des caractéristiques de transmission et de courant en fonction de la géométrie de la structure. Ces évolutions ont été vérifiées expérimentalement pour des doubles bar-

Tableau I. - Sens des variations de l'énergie de résonance, des caractéristiques de transmission et de courant en fonction de la géométrie et des paramètres matériaux.

[Trends for resonant energy, transmission and current characteristics versus geometry and material parameters.]

\begin{tabular}{|c|c|c|c|c|c|}
\hline & $\varepsilon_{\mathrm{r}}$ & $T_{\text {hors res }}$ & $T_{\text {rés }} / T_{\text {horrs }_{\text {ress }}}$ & $J_{\text {pic }}^{\prime}$ & $J_{\text {pic }} / J_{\text {vallé }}$ \\
\hline$L_{\mathrm{B}} \backslash$ & $\nu$ & $\nearrow$ & $\searrow$ & $\nearrow$ & $\searrow$ \\
\hline$\left.L_{\mathbf{w}}\right\rangle$ & $\pi$ & $\lambda$ & $\searrow$ & $\nearrow$ & $\searrow$ \\
\hline $\begin{array}{c}\Delta V \nearrow \\
T_{\mathrm{g}} T_{\mathrm{d}} \searrow\end{array}$ & $\nearrow$ & $\searrow$ & $\pi$ & $\searrow$ & $\nearrow$ \\
\hline $\begin{array}{l}m_{\text {balluere }}^{*} \\
T_{\mathrm{g}} T_{\mathrm{d}}\end{array}$ & $\nearrow$ & $\searrow$ & $\pi$ & $\searrow$ & $\pi$ \\
\hline
\end{tabular}


rières AlAs/GaAs [5, 20]. Nous avons fait figurer également les variations de ces mêmes grandeurs en fonction de la hauteur des barrières de potentiel $\Delta V$ ainsi que de la masse effective. Ces évolutions se comprennent facilement en considérant les implications des paramètres matériaux sur les transparences des barrières. Leur analyse va nous guider dans le choix d'un matériau.

\section{Choix d'un matériau et optimisation des structu- res.}

4.1 Comparaison. - Le tableau II donne les valeurs respectivement des masses effectives, de discontinuités de bande interdite et de bande de conduction pour les systèmes GaAlAs, GaInAsP, InGaAlAs. Concernant les structures GaAs/ GaAlAs, les masses effectives sont importantes, la discontinuité de bande de conduction limitée par le passage gap direct-gap indirect. Les performances en contraste et densité de courant sont dans ce cas modestes. Cependant, la croissance de ces matériaux est très bien maîtrisée et permet la réalisation de structures dissymétriques. Les doubles barrières GaAs/AlAs permettent théoriquement de limiter la contribution thermoionique du courant traversant la structure [5]. Les performances actuelles sont relativement modestes cependant. Leur interprétation pose le problème des effets tunnel dans les barrières de gap indirect [21]. Pour les matériaux incorporant du GaInAs, les masses effectives sont faibles. Il devrait être ainsi possible de polariser les structures à densités de courant élevé condition nécessaire à leur utilisation en très hautes fréquences. Les dou- bles barrières GaInAs / AlInAs permettent en outre de préserver un contraste de courant important compte tenu de la discontinuité de bande de conduction égale à $0,5[22,23]$.

4.2 OptimisAtion. - L'obtention de densité de courant de polarisation et de rapport courant $I_{\text {pic }}$ sur $I_{\text {vallé }}$ élevé sont deux objectifs antinomiques. Pour illustrer ce point, nous donnons sur la figure $10 a$ et $b$ les variations de la transmission en fonction de la tension collecteur appliquée respectivement pour deux épaisseurs de barrières $40 \AA$ et $30 \AA$. Les caractéristiques électriques sont données en Insert. Il s'agit ici d'une structure à double barrière AlInAs. On constate que le fait de diminuer l'épaisseur de barrière provoque une augmentation assez importante de la valeur pic du courant traversant la structure de $8 \mathrm{kA} / \mathrm{cm}^{2}$ à environ $40 \mathrm{kA} / \mathrm{cm}^{2}$. Ceci s'accompagne d'un élargissement de la courbe de transmission comparativement aux variations extrêmement sélectives de $T$ données figure $10 \mathrm{a}$.

S'il existe un compromis entre $J_{\text {pic }} / J_{\text {vallee }}$ et $J_{\text {pic }}$, il est possible toutefois d'optimiser les structures soit pour des applications à densité de courant importante, soit pour une recherche de la nonlinéarité la plus élevée possible. La figure 11 montre un exemple d'optimisation par variation de l'épaisseur des barrières en structure dissymétrique. La courbe en pointillé est obtenue lorsque les transmissions des barrières gauche et droite en polarisation sont parfaitement égales. $\mathrm{Au}$ vu de ces résultats on peut s'apercevoir qu'il n'est pas utopique d'avoir des courants de polarisation supérieurs à $50 \mathrm{kA} / \mathrm{cm}^{2}$ tout en conservant le caractère sélectif de la transmission.

Tableau II. - Paramètres matériaux.

[Material parameters.]

\begin{tabular}{|c|c|c|c|c|}
\hline Matériau & $m^{*}$ conduction & $\Delta \varepsilon_{\mathrm{g}}(\mathrm{eV})$ & $\Delta \varepsilon_{\mathrm{c}}(\mathrm{eV})$ & Remarque \\
\hline $\begin{array}{c}\text { GaAs/GaAlAs } \\
x=0,37\end{array}$ & $0,067 / 0,098$ & $\begin{array}{c}1,42 / 1,88 \\
\Delta \varepsilon_{\mathrm{g}}=0,46\end{array}$ & 0,3 & $\begin{array}{c}\Delta \varepsilon_{\mathrm{c}}=65 \% \varepsilon_{\mathrm{g}} \\
\text { density of state } \\
\text { effective mass }\end{array}$ \\
\hline GaAs/AlAs & $0,067 / 0,150$ & $\begin{array}{c}1,42 / 2,17 \\
\Delta \varepsilon_{\mathrm{g}}=0,75\end{array}$ & 0,49 & $\begin{array}{c}\text { Choix des masses effectives } \\
\text { et discontinuités } \\
\Gamma-X \text { et/ou } \Gamma-\Gamma\end{array}$ \\
\hline GaInAs/InP & $0,04 / 0,08$ & $\begin{array}{c}0,73 / 1,29 \\
\Delta \varepsilon_{\mathrm{g}}=0,56\end{array}$ & 0,2 à 0,4 & $\begin{array}{l}\text { Indétermination sur la valeur } \\
\text { des discontinuités : tunnel } \\
\text { de trous, tunnel d'électrons }\end{array}$ \\
\hline GaInAs/InAlAs & $0,04 / 0,0836$ & $\begin{array}{c}0,73 / 1,47 \\
\Delta \varepsilon_{\mathrm{g}}=0,74\end{array}$ & $\sim 0,5$ & 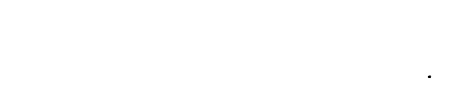 \\
\hline
\end{tabular}




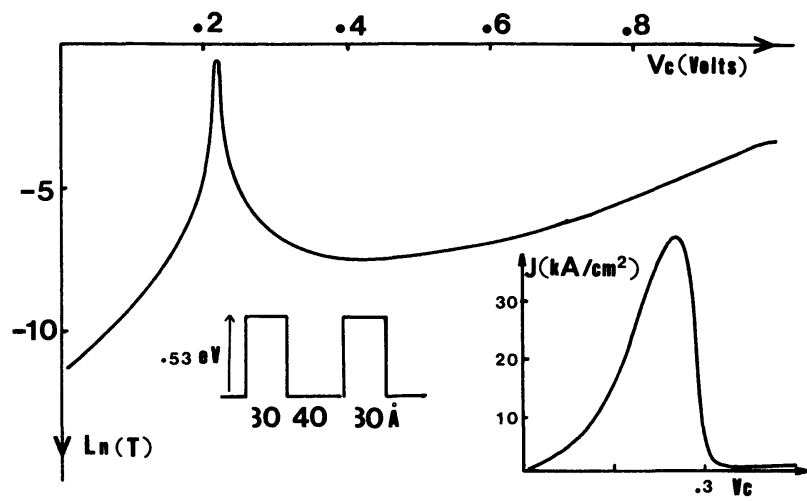

a)

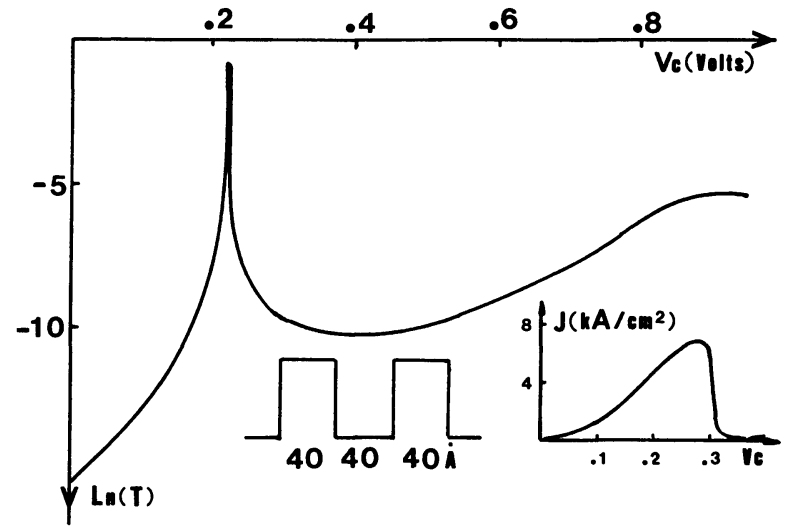

b)

Fig. 10. - Transmission et densité de courant en fonction de la tension appliquée (structure AlInAs/InGaAs / AlInAs).

[Transmission and current density versus the applied voltage (AlInAs/InGaAs DBH).]

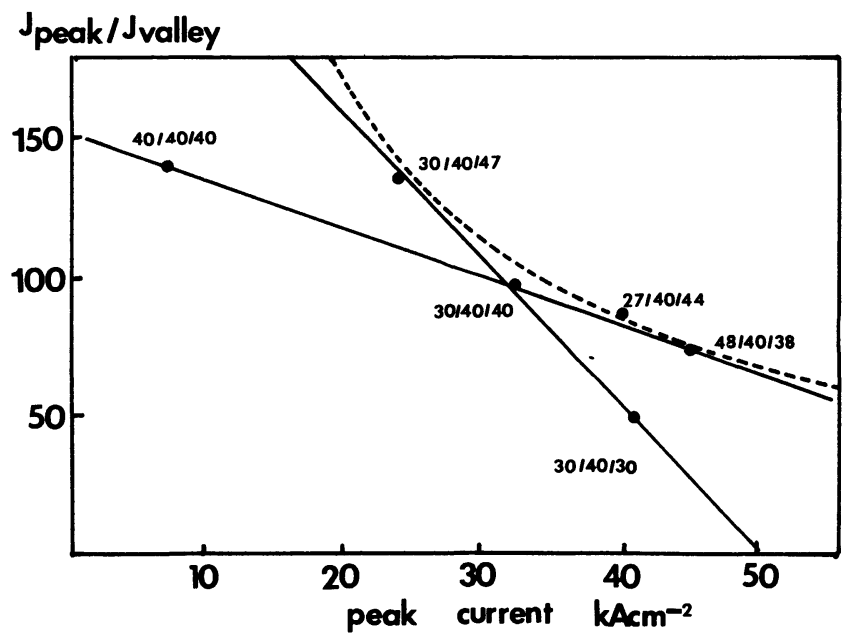

Fig. 11. - Optimisation par l'emploi de structures dissymétriques.

[Optimization by means of asymmetric resonant structures.]
5. Temps de réponse et réaction de charge d'espace.

5.1 TEMPS DE RÉPONSE. - Le temps de réponse des structures à tunnel résonnant fait actuellement l'objet de nombreuses études. Que ce soit, au plan théorique, la résolution de l'équation de Schrödinger dépendant du temps $[24,25]$ où, au plan expérimental, les caractérisations en photoluminescence résolue en temps [26]. Nous ne prétendons pas ici faire une analyse exhaustive de ce sujet mais plutôt d'essayer de voir les évolutions du temps de réponse en fonction de la géométrie de barrière et de préciser dans quelle mesure il est possible d'avoir un temps effectif inférieur à la picoseconde. Il est maintenant bien établi que l'inertie des structures à double barrière est dominée par le temps de piégeage des porteurs à l'intérieur du puits de potentiel [27]. Ce temps de résidence dans les états localisés dépend, par l'intermédiaire des barrières, du couplage avec le continuum formé par les zones de contact. Or, nous avons vu qu'un couplage plus ou moins lâche se traduit au niveau de la courbe de transmission par un élargissement plus ou moins important de la résonance. La largeur de résonance calculée en régime statique peut par conséquent constituer une première approximation du temps de réponse des structures à double barrière. Sur cette base, la figure 12 montre

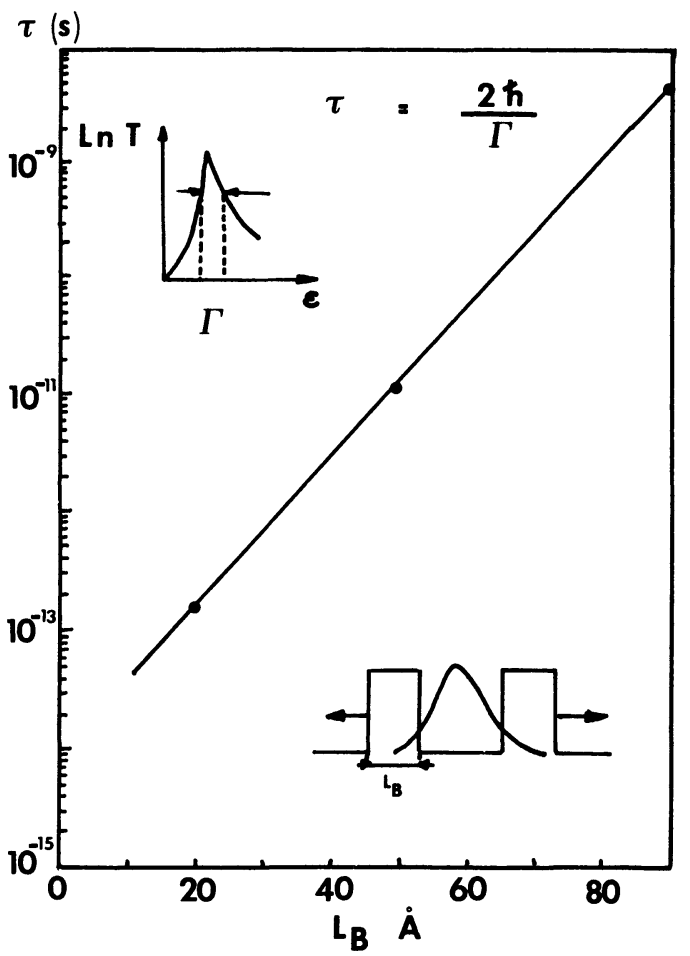

Fig. 12. - Temps de réponse évalué à partir de la largeur à mi-hauteur de la courbe de transmission.

[Response time calculated from full width at half maximum of the resonant level $(\mathrm{GaAlAs} / \mathrm{GaAs} x=0.4) L_{\mathrm{w}}=$ $50 \AA$. 
l'évolution du temps de réponse calculé à partir de la relation $\tau=2 \hbar / \Gamma$ ( $\Gamma$ largeur à mi-hauteur [28] respectivement pour $L_{\mathrm{B}}=20,50$ et $90 \AA$ ). Les largeurs à mi-hauteurs sont de $8,3 \times 10^{-3}, 1,02 \times$ $10^{-4}$ et $2,47 \times 10^{-7} \mathrm{eV}$. La structure test est une double barrière $\mathrm{Ga}_{0,63} \mathrm{Al}_{0,37} \mathrm{As} / \mathrm{GaAs}$. On constate que la recherche d'un faible temps de réponse impose des largeurs de barrières très réduites. Pour cet exemple, les temps estimés sont de 150 femtosecondes pour $L_{\mathrm{B}}$ de $20 \AA$. Les temps de passage par effet tunnel à travers les barrières sont estimés un ordre de grandeur inférieur à cette valeur [27].

5.2 RÉACTION DE CHARGE D'ESPACE. - L'autre problème essentiel dans les applications composants concerne la réaction de charge d'espace, produite par la charge piégée tant à l'intérieur des barrières que dans les zones adjacentes (zones de spacers [29, 32]). La façon dont la réaction de charge modifie le potentiel vu par les électrons peut se comprendre à partir de l'Insert de la figure 13 qui reprend l'approche initialement proposée par Ricco [15]. Celle-ci est basée sur la situation d'un puits infiniment étroit. La densité surfacique de charge piégée provoque une discontinuité au niveau du champ électrique interne et donc une rupture dans la variation spatiale du potentiel. Ceci a pour conséquence qu'il faut plus de tension pour réaliser la condition de résonance. Cet effet est par ailleurs accentué par l'accumulation de charge en amont de la structure à double barrière.

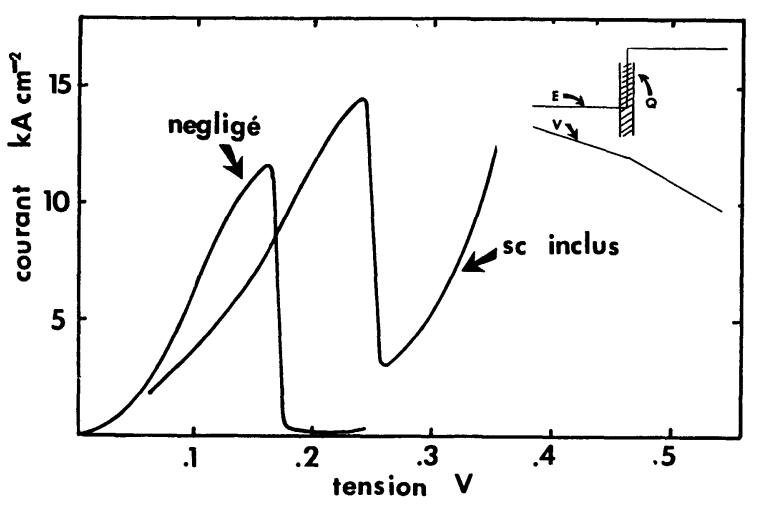

Fig. 13. - Influence de la réaction de charge d'espace. (a) variation spatiale du potentiel ; (b) caractéristique $I(V)$.

[Space charge reaction. (a) potential variation; (b) $I-V$ characteristic.]

Un résultat de calcul plus exact des variations spatiales de potentiel obtenu par résolution self consistante des équations de Schrödinger et de Poisson est donné figure 13a. La caractéristique $I(V)$ correspondante, comparée à celle obtenue sans réaction de charge d'espace est tracée figure $13 \mathrm{~b}$. On constate que l'augmentation de la tension de résonance s'accompagne d'une diminution très forte du rapport $I_{\text {pic }} / I_{\text {vallée }}$.

\section{Caractérisation physique et électrique.}

6.1 Physique. - La caractérisation des doubles barrières est tout d'abord difficile du fait des différences de structures entre les couches épitaxiées pour une application composant ou pour une étude physique. C'est ainsi le cas de la caractérisation des structures à tunnel résonnant par photoluminescence pour laquelle il est souvent nécessaire de concevoir des structures avec des barrières relativement larges et une couche épitaxiée en surface d'épaisseur faible, non dopée. Par contre, pour les structures destinées à être utilisées en composant il est impératif de minimiser $L_{\mathrm{B}}$ et de faire croître en surface une couche $\mathrm{N}^{+}$de quelques milliers d'angströms d'épaisseur de dopage supérieur à $10^{18}$ (réalisation du contact ohmique).

Ensuite, de nombreuses méthodes d'études utilisées très largement pour la caractérisation des hétérostructures multiples ne sont plus applicables ici en raison de l'absence de périodicité et du faible volume de semiconducteurs mis en jeu. C'est le cas des rayons $\mathrm{X}$ dont l'application au cas des multipuits quantiques permet de déterminer les paramètres structuraux (géométrie et composition d'alliage). En ce qui concerne les expérimentations de photoluminescence, outre la spécificité des structures évoquées ci-dessus, il apparaît souvent nécessaire d'utiliser les techniques de photoluminescence résolue en temps [26].

La figure $14 \mathrm{a}$ et $\mathrm{b}$ montre les résultats préliminaires de photoluminescence continue et résolue en temps pour une structure de géométrie $100 \AA / 50 \AA / 100 \AA$. La couche épitaxiée au centre hyperfréquence et semiconducteurs de Lille est schématisée sur cette figure. Les expérimentations de photoluminescence ont été effectuées par le CNET à $2 \mathrm{~K}$ en continu, à $45 \mathrm{~K}$ pour les intensités résolues en temps. On constate que pour des épaisseurs de barrière assez importantes il est possible d'accéder au niveau de confinement en raison de la localisation très importante des porteurs à l'intérieur du puits. Le niveau calculé est ici de $100 \mathrm{meV}$ valeur cohérente pour un puits de $50 \AA$.

Pour la photoluminescence résolue en temps, bien que l'on observe une décroissance quasi exponentielle de l'intensité en fonction du temps, l'interprétation de ces résultats est encore problématique. En effet, pour des largeurs de barrière aussi importantes il semble difficile d'accéder au temps de fuite à travers la barrière dont la valeur peut devenir du même ordre de grandeur que le temps de vie radiative. La détermination des niveaux d'énergie et de temps de durée de vie associés dans des conditions de faible localisation devrait permettre d'apporter 

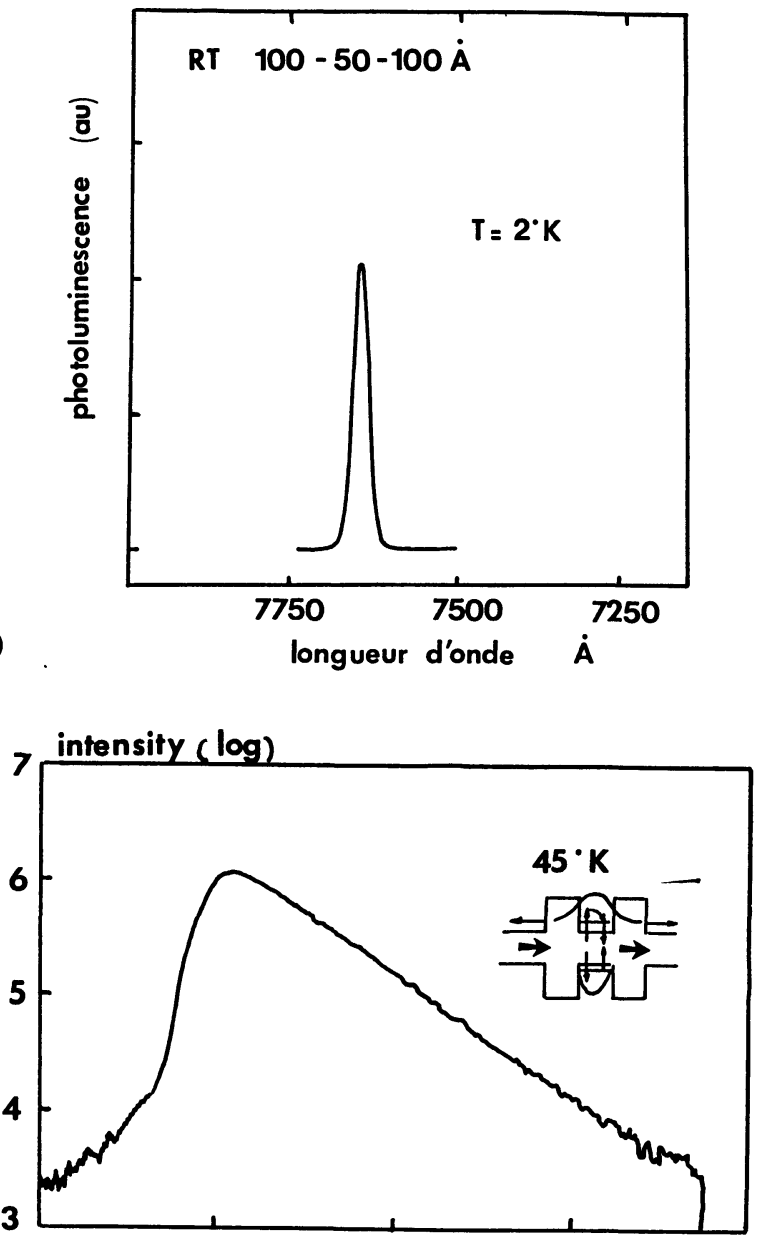

b)

temps 120ps $\mathrm{cm}^{-1}$

Fig. 14. - (a) Spectre de photoluminescence pour une structure GaAlAs $100 \AA / \mathrm{GaAs} 50 \AA / \mathrm{GaAlAs} 100 \AA$. (b) variation en fonction du temps.

[(a) Photoluminescence spectra for a GaAlAs $100 \AA / \mathrm{GaAs} 50 \AA / \mathrm{GaAlAs} 100 \AA$ structure. (b) time resolved PL measurement.]

des éléments d'informations sur la dynamique des structures à tunnel résonnant appliquées aux composants. D'autres méthodes d'analyse sont utilisées actuellement [34]. Elles demandent cependant d'accéder aux caractéristiques de conduction. Nous allons considérer ce point à présent.

6.2 CARACTÉRISTIQUES ÉLECTRIQUes. - La figure 15 montre un exemple de caractéristique statique visualisée directement sur traceur. Il s'agit d'une structure GaAs/AlGaAs (concentration en aluminium 0,3 ) servant à l'étude des fonctionnements à courant et tension élevés. Dans ce but, des couches GaAs relativement peu dopées ont été insérées dans la zone active. On constate que dans ce cas la tension de résonance est largement supérieure à la valeur théorique d'une double barrière simple, de l'ordre de $200 \mathrm{mV}$ pour une structure semblable.

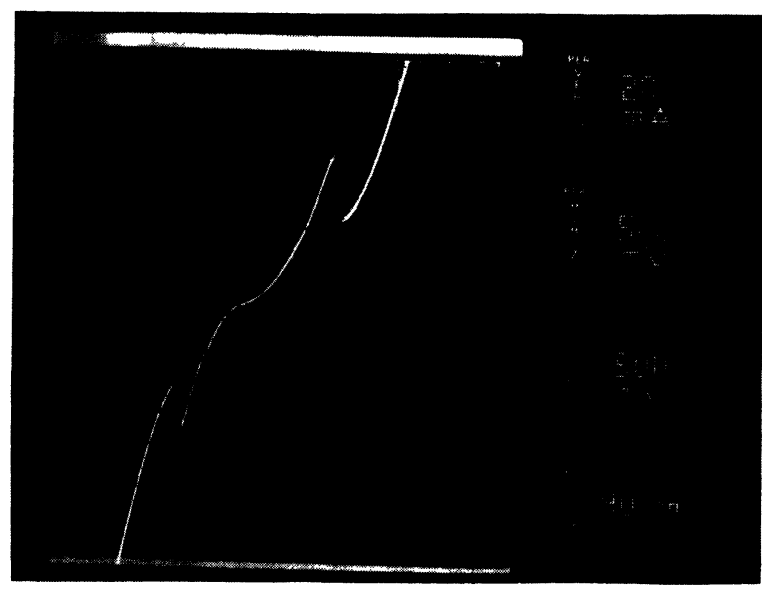

Fig. 15. - Caractéristique $I-V$ d'une structure GaAlAs / GaAs/GaAlAs mesurée à $296 \mathrm{~K}$ sur des échantillons avec $L_{\mathrm{B}}=L_{\mathrm{w}}=50 \AA$ (concentration en aluminium $30 \%$ ). Les zones de contact sont fortement dopées $N_{\mathrm{D}}=210^{18}$ et séparées de la zone active par des spacers peu dopés $\left(N_{\mathrm{D}}=210^{16}\right)$ de $75 \mathrm{~nm}$ d'épaisseur.

[Typical $I-V$ characteristics of AlGaAs/GaAs/AlGaAs resonant tunnelling measured at $296 \mathrm{~K}$ on samples with $L_{\mathrm{w}}=L_{\mathrm{B}}=50 \AA$ (aluminium concentration $30 \%$ ). Electrodes are heavily doped $N_{\mathrm{D}}=210^{18} \mathrm{~cm}^{-3}$ and separated from the double barrier region by low-doped GaAs $\left(N_{\mathrm{D}}=210^{16}\right)$ space layers of $75 \mathrm{~nm}$ in thickness.]

Le diamètre du mésa est de $15 \mu \mathrm{m}$. On constate par conséquent que ces structures admettent des densités de courant de polarisation, $J_{\text {pic }}$ de l'ordre de $35 \mathrm{kA} / \mathrm{cm}^{2}$ suffisamment importantes pour envisager une application très haute fréquence.

La figure 16 donne les modifications apportées sur la caractéristique $I(V)$ par un abaissement de la température à l'azote. La densité de courant pic est relativement peu modifiée. En revanche le rapport $J_{\text {pic }} / J_{\text {vallee }}$ initialement de 1,8 passe à 6,5 à $T=$ $77 \mathrm{~K}$. Ces résultats en statique sont comparables aux meilleures valeurs publiées dans la littérature pour cette concentration en aluminium [6]. Par ailleurs on note qu'aux deux températures, la diminution de courant dans la plage d'existence de la résistance négative se fait par paliers au nombre de 2 à $77 \mathrm{~K}$. La prise en compte des niveaux d'énergie discrets du puits créé en amont de la première barrière en polarisation devrait permettre de décrire ces effets théoriquement [35].

Outre les résultats de conductance différentielle négative, l'étude de la caractéristique $I(V)$ en fonction de la température permet d'apporter d'autres éléments d'informations sur les structures à tunnel résonnant. Ainsi, il a été montré très récemment que la détermination des variations du courant en fonction de la tension avant résonance peut permettre de déterminer l'énergie de confinement dans le puits [36]. Enfin, un certain nombre d'études sous champ magnétique [35] et sous pression [36] ont 


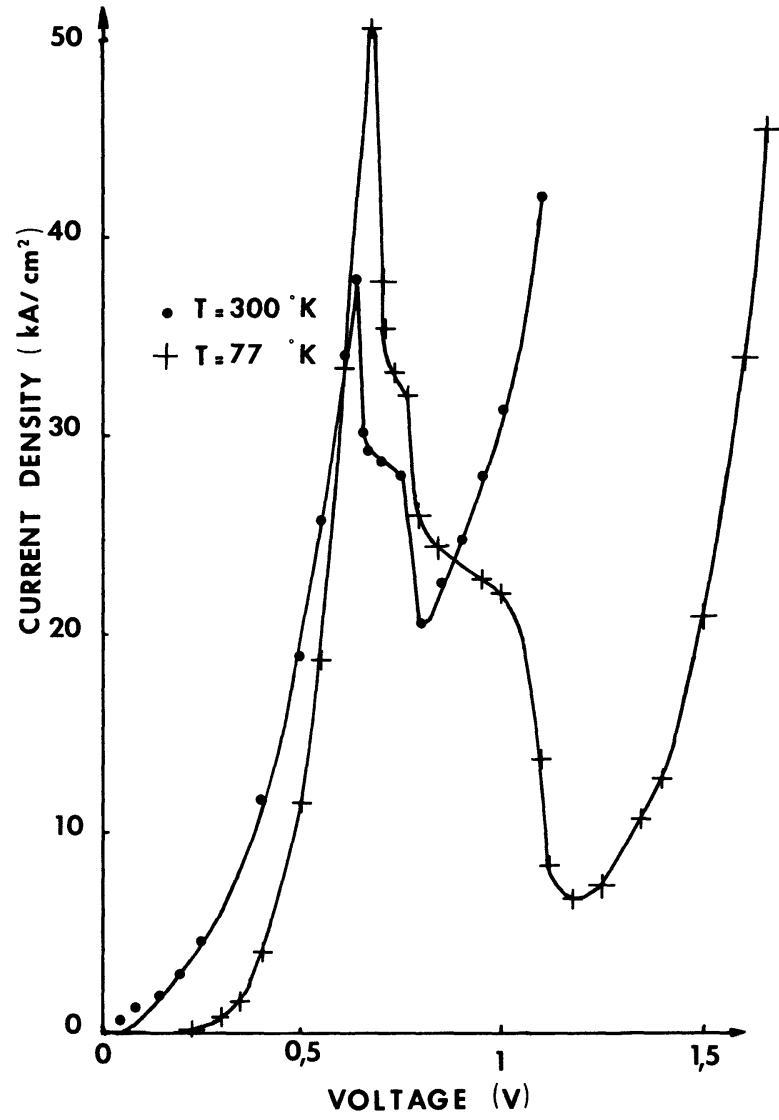

Fig. 16. - Caractéristiques $I-V$ à $300 \mathrm{~K}$ et $77 \mathrm{~K}$.

[I-V characteristic at room and nitrogen temperatures.]

été entreprises. Il a été en particulier démontré que par magnétotransport on accède aux variations de l'énergie du puits en fonction de la tension appliquée pour des structures avec une zone non dopée dans l'émetteur et le collecteur. Quant aux caractérisations sous pression hydrostatique, il devrait être possible de préciser le rôle joué par les minimums d'énergie d'ordre supérieur dans la bande de conduction.

\section{Applications composants.}

Nous donnons sur la figure 17 les différentes filières composants intégrant des structures à double barrière. Pour les dipôles on peut distinguer les diodes tirant parti uniquement de l'effet tunnel résonnant, des composants à injection et temps de transit. Pour les tripôles, il est relativement difficile d'établir des classes dans la mesure où les composants diffèrent par leurs structures (verticale ou planar) ainsi que par la nature des porteurs assurant la conduction (unipolaire et bipolaire). Nous avons également mentionné pour mémoire le cas des structures à simple barrière souvent à la base des structures utilisant l'effet tunnel résonnant. C'est le cas du transistor à électrons chauds [37] dont le RHET est le prolongement.

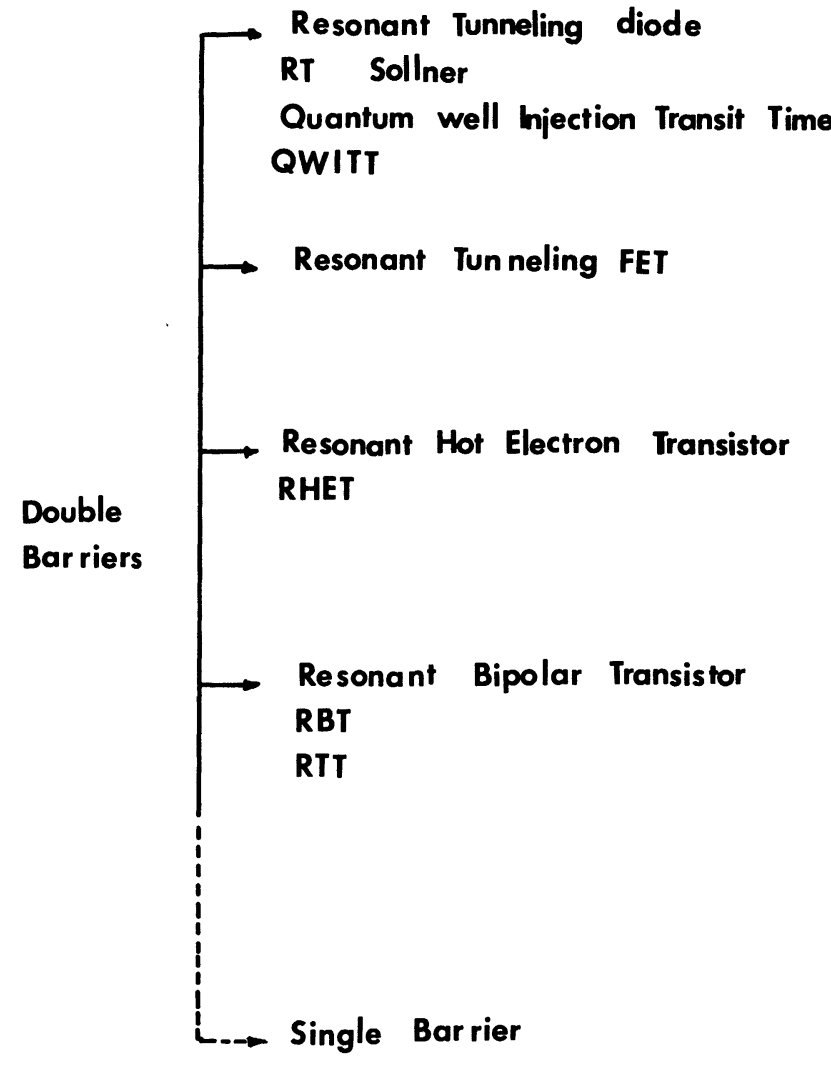

Fig. 17. - Classification des composants à tunnel résonnant.

[Classification of resonant tunnelling devices.]

Bien que très disparate dans leur classification, l'ensemble de ces composants ont en commun le fait que les effets de tunnel résonnant sont très souvent indissociables des effets d'électrons chauds. La motivation de recherche de ces derniers effets est simple. Il s'agit en effet de ne pas trop augmenter le temps de réponse global du composant du fait de l'inertie au transit des porteurs. Nous allons considérer quelques exemples d'applications à présent.

7.1 UTILISATION EN DIPÓLE. - La plupart des applications utilisant l'effet tunnel résonnant en dipôle concernent la réalisation d'oscillateurs millimétriques. Si l'on considère en effet les oscillateurs plus conventionnels à l'état solide (diodes GUNNIMPATT) on peut montrer que leurs limitations fréquentielles se situent environ vers $140 \mathrm{GHz}$ pour les diodes GUNN et $200 \mathrm{GHz}$ pour les diodes IMPATT fonctionnant en mode fondamental [38, 39].

Des structures et modes de fonctionnement nouveaux ont été depuis proposés [41, 42]. Leur aptitude à monter en fréquence reste cependant à démontrer. Par contre, des résultats extrêmement encourageants ont été obtenus à $200 \mathrm{GHz}$ avec une structure double barrière GaAs/AlAs [43] complétant les résultats en détection évoqués dans l'introduction. 
En contrepartie à cet avantage fréquentiel, le fonctionnement des structures à tunnel résonnant se fait à tension très faible. Il en résulte que les excursions de tensions alternatives donc les puissances de sortie sont également très faibles pour des structures simples à double barrière. Le fait d'adjoindre à la structure de base à puits quantique une zone de transit peut permettre de pallier cet inconvénient. Les effets de transit peuvent devenir dans ce cas prépondérants, la structure résonnante jouant le rôle d'une zone d'émission. La structure QWITT (Quantum Well Injection Transit Time) est basée sur ce principe [44]. Cependant, son mode de fonctionnement peut, par déplacement du point de polarisation, différer de celui de la diode à tunnel résonnant utilisé jusqu'à présent en oscillateur. Pour comprendre ces différences nous avons représenté figure 18 respectivement l'allure de la caractéristique $I(V)$ ainsi que celle du courant injecté par tunnel résonnant dans la zone de transit. La tension de polarisation est ici située en dehors de la plage d'existence de la résistance différentielle négative. La tension alternative $V_{\mathrm{RF}}$ provoque alors une augmentation très brutale des valeurs de courant en phase avec la tension. On constate par conséquent qu'en décalant le point de polarisation, il est possible de moduler la phase d'injection et d'obtenir une conductivité d'injection positive ou négative. Par
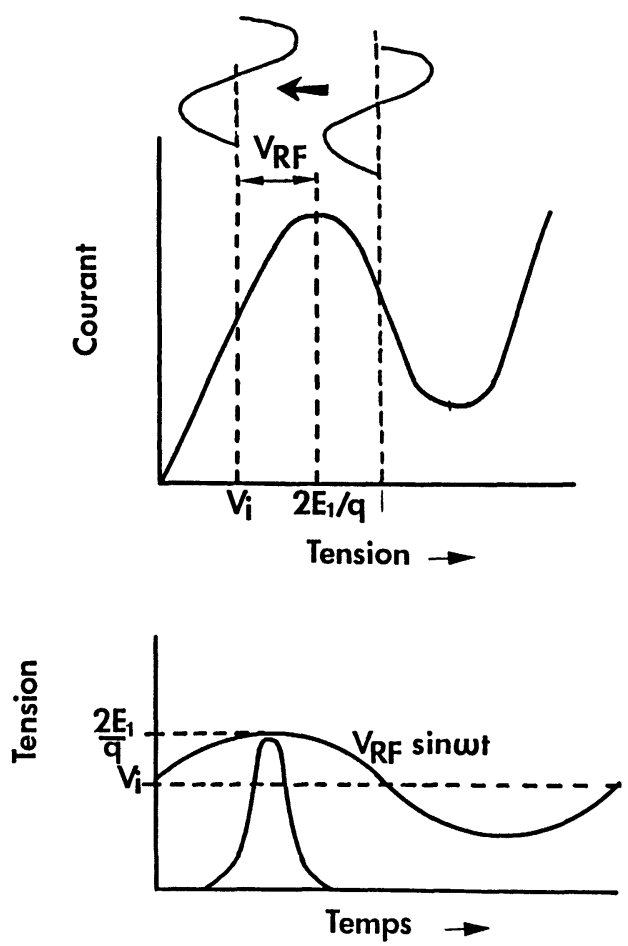

Fig. 18. - Principe de fonctionnement des structures QWITT (modulation de la phase d'injection).

[Operating principles of QWITT (modulation of injection phaseshift.] ailleurs le transport des porteurs dans la zone de transit est fortement non stationnaire car ils sont injectés à énergie relativement faible alors que le champ interne est élevé. Des phénomènes de survitesse peuvent alors se produire limitant ainsi le temps de transit.

7.2 RT FET. - Un exemple de structure à tunnel résonnant intégré dans un transistor FET [45] est donné sur la figure 19. La double barrière est pour cet exemple implantée sous la source. L'insertion sous la grille a été également proposée récemment [46]. L'un des buts des études sur ces structures est le suivant : il s'agit de faire varier grâce à l'adjonction d'une troisième électrode, la tension où apparaît la résistance différentielle négative. Le principe de cette modulation peut se comprendre sachant que la tension totale se décompose en la chute de tension aux bornes de la double barrière et celle du canal. Lorsque la résistance du canal change, différentes valeurs de tension totale doivent être appliquées pour satisfaire à la condition de résonance. De nombreux exemples de caractéristiques expérimentales sont donnés dans la référence [45]. Ainsi pour une structure $\mathrm{Al}_{0,45} \mathrm{Ga}_{0,55} \mathrm{As} / \mathrm{GaAs}$ il est possible de faire varier la tension de résonance de $200 \mathrm{mV}$ à $800 \mathrm{mV}$ en polarisant la tension de grille de $+0,4 \mathrm{~V}$ à $-1 \mathrm{~V}$. Néanmoins, le désavantage majeur de ce type de structure est que les limitations fréquentielles sont imposées par le transistor à effet de champ. Les composants FET à tunnel résonnant devraient donc être limités en fréquence.

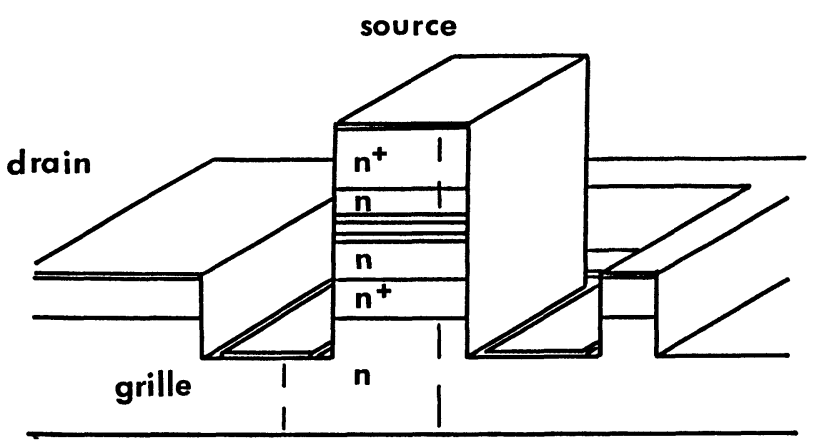

Fig. 19. - Structure du RT FET.

[Schematic cut away view of the RT FET.]

7.3 RHET. - La structure de base d'un composant RHET (Resonant Tunneling Hot Electron Transistor), réalisé en matériau GaAs/GaAlAs pour cet exemple, est schématisée sur la figure 20 . L'émetteur, la base et le collecteur sont de type n. Une double barrière est insérée entre émetteur et base, une barrière $\mathrm{AlGaAs}$ entre base et collecteur. Le principe du fonctionnement peut se comprendre à partir de celui de la structure tunnel résonnant, 

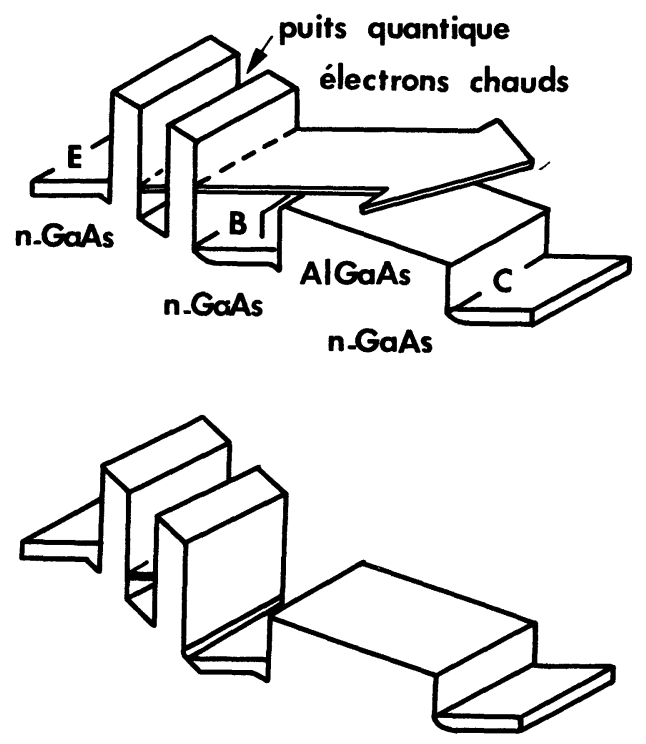

Fig. 20. - Structure du RHET.

[Schematic cross section of the RHET.]

utilisée également dans cette application en injecteur. Les caractéristiques courant collecteur courant de base en fonction de la tension base émetteur montrent une décroissance brutale des valeurs de courant lorsque le niveau d'énergie de la bande de conduction de l'émetteur devient supérieur à celui de l'état résonnant. On obtient dans ce cas une transconductance négative. Le temps de réponse effectif des structures RHET est estimé à des valeurs inférieures à la picoseconde compte tenu que les porteurs sont injectés dans la base avec une énergie cinétique importante. Pour des épaisseurs de base comprises entre 350 et $1000 \AA$, le transit des porteurs dans la base se fait de façon quasibalistique. Par ailleurs, l'optimisation des structures utilisant les effets conjugués de tunnel résonnant et d'électrons chauds fait intervenir d'autres critères que ceux évoqués aux paragraphes précédents. En particulier il est impératif de limiter l'énergie d'injection dans la base, fixée par celle de l'état résonnant, à une valeur inférieure à la différence d'énergie vallée centralevallée latérale. Dans le cas contraire en effet, le déclenchement des transferts intervallées provoque une forte augmentation des fréquences de collision. Cette énergie est de l'ordre de 0,3 pour le $\mathrm{GaAs}$, de 0,5 pour le GaInAs. L'utilisation de ce dernier matériau se révèle donc très intéressante si l'on veut concilier une optimisation des effets tunnel résonnant et d'électrons chauds. Les applications pratiques sont essentiellement logiques. Elles visent en particulier la réalisation de circuits élémentaires (NOR exclusif par exemple) avec un seul composant actif facilitant ainsi l'intégration [48, 49].

7.4 RBT. - La structure d'un RBT est donnée figure 21. Ce composant dérive du même principe

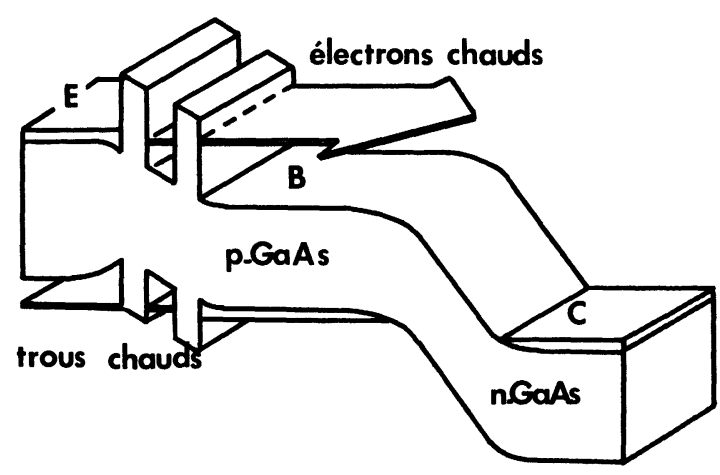

Fig. 21. - Structure du RBT.

[Schematic cross section of the RBT.]

que le RHET. La différence vient que le RBT utilise une base de type $\mathrm{p}$. Il n'y a donc pas de barrière dans le collecteur. Il en résulte que le RBT a un gain en courant plus élevé car les porteurs chauds peuvent atteindre le collecteur même lorsqu'ils perdent de l'énergie cinétique au cours de leur transport dans la base. En outre, des trous sont injectés de la base vers l'émetteur par tunnel résonnant. Le flux de trous contribue au courant de base, le flux d'électrons au courant collecteur. Ainsi par observation du courant de base et de collecteur en fonction de la tension base émetteur il est possible de mettre en évidence le tunnel des électrons puis des trous. Le RBT est utilisé essentiellement en logique [50].

\section{Conclusion.}

$\mathrm{Au}$ terme de cette présentation, les points suivants nous semblent devoir être rappelés.

Pour l'aspect modélisation, il est tout d'abord concevable de décrire avec une assez bonne approximation les caractéristiques électriques à partir de modèles simplifiés. Des études systématiques sont alors possibles. A titre d'exemple, nous avons proposé une optimisation en contraste et courant pic de structure à double barrière AlInAs/GaInAs. Par contre, lorsque les structures sont utilisées en composant, il est souvent nécessaire de travailler à densités de courant élevées et de prendre en compte des zones actives étendues. Des modèles plus exacts sont alors nécessaires. Un exemple d'application à l'étude de la réaction de charge d'espace a été proposé ici à partir de la résolution consistante de l'équation de Schrödinger.

Pour l'aspect caractérisation physique et électrique, il est relativement difficile d'accéder directement aux informations traduisant l'état de confinement et sa durée de vie. Des résultats préliminaires de photoluminescence et des caractéristiques $I(V)$ ont été présentés. L'analyse fine de ces résultats confrontés à ceux obtenus par d'autres méthodes 
magnétotransport, en outre, devrait permettre de lever certaines ambiguïtés qui subsistent concernant les phénomènes physiques mis en jeu.

Pour l'aspect composant, de nombreuses idées nouvelles ont été publiées dans la littérature. Nous nous sommes efforcés de faire le point sur ce sujet. En guise de conclusion, nous retiendrons que les effets d'électrons chauds peuvent être marqués. Le transport à vitesse élevée qui en résulte permet dans la plupart des cas de limiter les inerties de transit. Ces effets introduisent en contrepartie des règles supplémentaires de conception dont il faut tenir compte.
De nombreux points relatifs au tunnel résonnant sont encore sujets à controverse, mal connus ou totalement éludés. A titre d'exemple citons le tunnel séquentiel $[28,18]$, le temps de réponse, les propriétés de bruit.

\section{Remerciements.}

Nous remercions B. Lambert et B. Deveaud du CNET B. Vinter, A. Tardella et D. Thomas du laboratoire central de recherche Thomson Corbeville, J. L. Lorriaux, Y. Druelle, A. Fattorini, E. Constant du centre hyperfréquences et semiconducteurs de Lille.

\section{Bibliographie}

[1] Tsu, R. and Esaki, L., Appl. Phys. Lett. 11 (1973) 562.

[2] Chang, L. L., Esaki, L., Tsu, R., Appl. Phys. Lett. 12 (1974) 593.

[3] Sollner, T. C. L. G. et al., Appl. Phys. Lett. 49 (1983) 588.

[4] TsuchiYa, M., SaKaKi, H. et Yoshino, T., Jpn J. Appl. Phys. 24 (1985) L466.

[5] Tsuchiya, M. et Sakaki, H., Appl. Phys. Lett. 49 (1986) 88.

[6] Huang, C. I. et al., Appl. Phys. Lett. 51 (1987) 121.

[7] InAta, T. et al., Jpn J. Appl. Phys. 25 (1986) L983.

[8] SEN, L. et al., Electron. Lett. 23 (1987) 1230.

[9] InAtA, T. et al., Jpn J. Appl. Phys. 26 (1987) 1332.

[10] Brown, E. R. et al., Appl. Phys. Lett. 50 (1987) 83.

[11] GRÖNQVIST, H. et al., conference EuMC.

[12] SEN, S. et al., IEEE Trans. Electron Dev. ED-34 (1987) 2185.

[13] Yokoyama, N. et al., Jpn J. Appl. Phys. 24 (1985) L853.

[14] Kane, E. O., Tunneling Phenomena in solids, Eds E. Burstein et S. Lundquist (Plenum Press) 1969.

[15] Ricco, B. et Azbeil, M. Ya., Phys. Rev. B 29 (1984) 1970.

[16] Vassel, M. O., Johnson, L. et Lockwood, J. Appl. Phys. 54 (1983) 5206.

[17] Fromhold, A. T., Quantum Mechanics for Applied Physics and Engineering (Academic press) 1981.

[18] Weil, T. et Vinter, B., Appl. Phys. Lett. 50 (1987) 1281.

[19] BAdher, T. B., Morrison, C. A. et Bruno, J. D., Appl. Phys. Lett. 51 (1987) 1089.

[20] Tsuchiya, M. et SaKaKI, H., Jpn J. Appl. Phys. 25 (1986) L185.

[21] Brey, L. et Tejedor, C., Solid State Commun. 61 (1987) 573.

[22] Sug Iyama et al., Jpn J. Appl. Phys. 25 (1986) L983.

[23] InAta, T. et al., Jpn J. Appl. Phys. 25 (1986) L983.

[24] Jauho, A. P. et NiETo, M. M., Superlattices and microstructures 2 (1986) 407.

[25] JogAI, B. et WANG, K. L., Superlattices and microstructures 2 (1986) 259.

[26] Tsuchiya, M., Matsusue, T. et Sakaki, H., Phys. Rev. Lett. 59 (1987) 2356.
[27] KlluKSDahl, N. C. et al., Solid State Electron. 31 (1988) 743

[28] Jonson, M. et Grincwajg, A., Appl. Phys. Lett. 51 (1987) 1729.

[29] Cahay, M. et al., Appl. Phys. Lett. 50 (1987) 612.

[30] Ohnishi, et al., Appl. Phys. Lett. 49 (1986) 1248.

[31] Brennan, K. F., J. Appl. Phys. 62 (1987) 2392.

[32] Payne, M. C., Semicond. Sci. Technol. 2 (1987) 797.

[33] Goldman, V. J., Tsui, D. C., Phys. Rev. B 35 (1987) 9387.

[34] Payling, C. A. et al., Surface Science (North Holland, Amsterdam), à paraître.

[35] Goldman, V. J., Tsui, D. C. et Cunningham, J. E., Phys. Rev. Lett. 58 (1987) 1256.

[36] Hughes, O. H. et al., à paraître dans J. Vac. Sci. Techn.

[37] Heiblum, M., Solid State Electron. 24 (1981) 343.

[38] LipPENS, D., NiERUChalsKi, J. L. et Constant, E., IEEE Trans. Electron Dev. ED-32 (1985) 2269.

[39] LipPENS, D., Friscourt, M. R., Rolland, P. A. et Crosnier, Y., Rev. Phys. Appl. 22 (1987) 1433.

[40] Friscourt, M. R., Rolland, P. A. et Pernisek, M., IEEE Electron. Dev. Lett. EDL-6 (1985) 497.

[41] Lippens, D., Nieruchalski, J. L., Appl. Phys. Lett. 48 (1986) 1464.

[42] Hess, K., Solid State Electron. 31 (1988) 319.

[43] Brown, E. R., Sollner, T. G. L. G., Goddhue, W. D. et PARKER, C. D., IEEE Trans. Electron. Dev. ED-34 (1987) 2381.

[44] Kesan, V., Neikirk, D. P., Streetman, S. G. et Blakey, P., IEEE Electron Dev. Lett. EDL-8 (1987) 129.

[45] Woodward, T. K., McGill, T. C., ChUnG, H. F., Burnham, R. D., Appl. Phys. Lett. 51 (1987) 1542.

[46] Sen, S., Capasso, F., Beltram, F. et Cho, A., IEEE Trans. Electron Dev. ED-34 (1987) 1768.

[47] WoODWARD, T. K. et al., IEEE Electron Dev. 9 (1988) 122.

[48] YoKoy ama et al., Solid State Electron. 31 (1988) 577.

[49] IMAMURA et al., IEEE Trans. Electron Dev. ED-34 (1987) 2382.

[50] Futatsugi et al., Jpn J. Appl. Phys. 26 (1987) 2131. 